\author{
Article \\ Doi 10.5943/sif/1/1/8 \\ Copyright (C) Mushroom Research Foundation 2016
}

\title{
Camarosporium uniseriatum nom. nov., from Celtis occidentalis in European Russia
}

\section{Thambugala KM ${ }^{1,2,3}$, Bulgakov $\mathrm{TS}^{4}$, Eungwanichayapant $\mathrm{PD}^{3}$, Liu $\mathrm{ZY}^{1}$ and Hyde $\mathrm{KD}^{2,3}$}

${ }^{1}$ Guizhou Key Laboratory of Agricultural Biotechnology, Guizhou Academy of Agricultural Sciences, Guiyang 550006, Guizhou, People's Republic of China

${ }^{2}$ Center of Excellence in Fungal Research, Mae FahLuang University, Chiang Rai 57100, Thailand

${ }^{3}$ School of Science, Mae Fah Luang University, Chiang Rai 57100, Thailand

${ }^{4}$ Academy of Biology and Biotechnology, Southern Federal University, Rostov-on-Don 344090, Rostov region, Russia

Thambugala KM, Bulgakov TS, Eungwanichayapant PD, Liu ZY, Hyde KD 2016 Camarosporium uniseriatum nom. nov., from Celtis occidentalis in European Russia. Studies in Fungi 1(1), 90-98, Doi 10.5943/sif/1/1/8

\begin{abstract}
Celtis occidentalis (American hackberry) is a deciduous tree widely distributed in northern America and introduced in many regions of Europe. In this study we collected Cucurbitaria celtidis from dead or dying twigs and branches of $C$. occidentalis (Cannabaceae) in the Rostov region (Southern European Russia), where this tree is a common ergasiophyte in artificial forests. The placement of this species in Camarosporium sensu stricto in Pleosporinae, Pleosporales is shown in a multi-locus tree based on combined LSU, SSU and ITS sequence data. Camarosporium uniseriatum nom. nov. is introduced based on morphological and phylogenetic analyses.
\end{abstract}

Keywords - Cucurbitaria - phylogeny - Pleosporales - sexual morph

\section{Introduction}

Camarosporium is a frequently accounted coelomycetous genus which was introduced by Schulzer (1870). Subsequently, during the past century, more than 500 epithets were recorded for this genus (Index Fungorum 2016). Recent studies based on multi-locus phylogeny showed that camarosporium-like species are polyphyletic in Pleosporales (Liu et al. 2015, Wijayawardene et al. 2014 a, b, 2015, 2016, Ariyawansa et al. 2015), and cucurbitaria-like sexual morphs have been reported for the genus (Wijayawardene et al. 2014b, Tibpromma et al. 2016). Wijayawardene et al. (2014b) established Camarosporium sensu stricto in Pleosporinae, Pleosporales with $C$. quaternatum Schulzer, the type of the genus, C. Aloes Crous \& M.J. Wingf. and two other new species. Liu et al. (2015) introduced three new Camarosporium species in Camarosporium sensu stricto.

Camarosporium elongatum and C. Arezzoensis Tibpromma, et al. are the sexual morphs reported for the genus Camarosporium and they share similar morphological characters with Cucurbitaria species (Doilom et al. 2013, Wijayawardene et al. 2014a, Tibpromma et al. 2016). In this study, we collected Cucurbitaria celtidis Shear on dead or dying twigs and branches of Celtis occidentalis in Russia, Rostov region (Southern European Russia), where this tree is a common ergasiophyte in artificial forests in steppe zone of Eastern Europe. We used morphological 
characters plus multi-gene molecular analyses to resolve its correct placement according to the modern taxonomic concepts.

\section{Materials and methods}

\section{Collection of samples, isolation and morphological examination}

Fresh specimens were collected from dead or dying twigs and thin branches of Celtis occidentalis in the Botanical Garden of Southern Federal University (Russia, Rostov region, Rostov-on-Don City) and samples were grown on potato dextrose agar. Isolates were derived via single spore isolation following the protocols of Chomnunti et al. (2014). Germinating spores were transferred to PDA and incubated at $25^{\circ} \mathrm{C}$ in the dark. Cultural characteristics, such as mycelium colour, shape, texture and growth rate were determined. Type specimens are deposited in the herbarium of Mae Fah Luang University (MFLU), Chiang Rai, and Guizhou Academy of Agricultural Sciences (GZAAS), while cultures are deposited at the Mae Fah Luang University Culture Collection (MFLUCC) with duplicates in International Collection of Microorganisms from Plants (ICMP) Landcare Research, New Zealand.

The specimens were observed and examined with a Motic SMZ 168 stereomicroscope. Micro-morphological characters of the specimen were examined under a Nikon ECLIPSE 80i compound microscope and images were captured using a Nikon ECLIPSE 80i compound microscope with a Canon EOS 550D digital camera. Observations and photographs were made from material mounted in water and Indian ink was added to water mounts to show the presence of gelatinous sheaths around the ascospores. Measurements were made with the Tarosoft (R) Image Frame Work and images used for figures were processed with Adobe Photoshop CS3 Extended version 10.0 software. Faces of fungi numbers and Index Fungorum numbers are provided as detailed in Jayasiri et al. (2015) and Index Fungorum (2016).

\section{DNA extraction, PCR amplification and sequencing}

Fresh fungal mycelium was grown on PDA at $25^{\circ} \mathrm{C}$ for 14 days. Extraction of genomic DNA from mycelia and sequencing of PCR products were carried out following the method of Thambugala et al. (2015). Four partial gene portions were amplified in this study including LROR and LR5 (Vilgalys \& Hester, 1990) for the nuclear ribosomal large subunit (LSU), ITS4 and ITS5 (White et al. 1990) for the internal transcribed spacer (ITS), EF1-983F and EF1-2218R (Carbone and Kohn 1999) for translation elongation factor 1-alpha (EF1- $\alpha$ ) and NS1 and NS4 (White et al. 1990) for the nuclear ribosomal small sub unit (SSU). The amplifications were performed in $25 \mu \mathrm{L}$

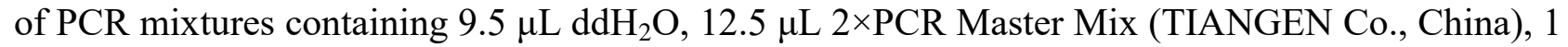
$\mu \mathrm{L}$ of DNA template, $1 \mu \mathrm{L}$ of each primer $(10 \mu \mathrm{M})$. The amplification reactions were performed and analysed as described by Thambugala et al. (2015).

\section{Phylogenetic analyses}

The phylogeny of the new strain was determined based on a combined data set of LSU, SSU and ITS sequence data of 44 isolates belonging to the suborder Pleosporineae, Pleosporales (Table 1) with Halojulella avicenniae (BCC 20173) as the out group taxon. SeqMan v. 7.0.0 (DNASTAR, Madison, WI) was used to compute consensus sequences. The sequence data were aligned and combined using Bioedit (Hall 1999) and MEGA 5.0 (Tamura et al. 2011). Phylogenetic analyses were performed based on maximum likelihood (ML) criterion using RAxML-HPC BlackBox (8.2.4) (Stamatakis et al. 2008) in the CIPRES (Miller et al. 2010). The general time reversible model of evolution including estimation of invariable sites (GTRGAMMA + I) and assuming a discrete gamma distribution with four rate categories was used for the ML analysis. The best scoring tree (-13523.550799) was selected and visualized with MEGA v. 5 (Tamura et al. 2011). ML Bootstrap support (BS) (greater than or equal $50 \%$ ) are shown below or above each branch (Fig. 1). All the sequences newly generated in this study are deposited in GenBank. 
Table 1 GenBank and culture collection accession numbers of species included in the phylogenetic study. Newly generated sequences are shown in bold.

\begin{tabular}{|c|c|c|c|c|c|}
\hline Species & Isolate no. & & $\begin{array}{l}\text { GenBank } \\
\text { Accession no }\end{array}$ & & \\
\hline & & LSU & SSU & ITS & $\overline{E F}$ \\
\hline Alternaria alternata & CBS 916.96 & DQ678082 & KC584507 & FJ196306 & \\
\hline Alternaria murispora & MFLU 14-0758 & KP334704 & KP334724 & KP334714 & \\
\hline Ascochyta pisi & CBS 126.54 & DQ678070 & DQ678018 & GU237772 & \\
\hline Bipolaris melinidis & BRIP 12897 & JN600994 & - & JX256411 & \\
\hline Camarosporium aborescentis & MFLUCC 14-0604 & KP711378 & KP711379 & KP711377 & \\
\hline Camarosporium aloes & CBS 136437 & KF777198 & - & KF777142 & \\
\hline Camarosporium arezzoensis & MFLUCC 14-0238 & KP120927 & KP120928 & KP120926 & \\
\hline Camarosporium aureum & MFLUCC 14-0620 & KP744478 & KP753948 & KP744436 & \\
\hline Camarosporium caraganicola & MFLUCC 14-0605 & KP711381 & KP711382 & KP711380 & \\
\hline Camarosporium uniseriatum & MFLUCC 15-0444 & KU697614 & KU697615 & KU697613 & KU697612 \\
\hline Camarosporium clematidis & MFLUCC 13-0336 & KJ562188 & KJ589414 & KJ562213 & \\
\hline Camarosporium quaternatum & CBS 483.95 & GU301806 & GU296141 & - & \\
\hline Camarosporium robiniicola & MFLUCC 13-0527 & KJ589412 & KJ589415 & KJ562214 & \\
\hline Camarosporium spartii & MFLUCC 13-0548 & KJ589413 & KJ589416 & KJ562215 & \\
\hline Comoclathris compressa & CBS 156.53 & KC584372 & KC584630 & - & \\
\hline Comoclathris sedi & MFLUCC 13-0817 & KP334705 & KP334725 & KP334715 & \\
\hline Coniothyrium palmarum & CBS 758.73 & EU754154 & EU754055 & _- & \\
\hline Coniothyrium palmarum & CBS 400.71 & EU754153 & EU754054 & AY720708 & \\
\hline Cucurbitaria berberidis & CBS 363.93 & GQ387606 & GQ387545 & JF740191 & \\
\hline Cucurbitaria berberidis & CBS 394.84 & GQ387605 & GQ387544 & - & \\
\hline Cucurbitaria elongata & MFLUCC 14-0260 & KJ724249 & - & - & \\
\hline Curvularia lunata & CBS 730.96 & JX256396 & _ & JX256429 & \\
\hline Didymella exigua & CBS 183.55 & EU754155 & GU296147 & GU237794 & \\
\hline Dothidotthia aspera & СРC 12933 & EU673276 & EU673228 & _ & \\
\hline Dothidotthia symphoricarpi & СРC 12929 & EU673273 & EU673224 & - & \\
\hline Halojulella avicenniae & BCC 20173 & GU371822 & GU371830 & - & \\
\hline Leptosphaeria doliolum & CBS 541.66 & JF740284 & - & JF740206 & \\
\hline Leptosphaeria doliolum & MFLUCC 13-0740 & KP729445 & _- & KP729444 & \\
\hline Leptosphaeria etheridgei & CBS 125980 & JF740291 & _- & JF740221 & \\
\hline Leptosphaerulina australis & CBS 317.83 & EU754166 & EU754067 & GU237829 & \\
\hline Neocamarosporium betae & CBS 109410 & EU754178 & EU754079 & - & \\
\hline Neocamarosporium goegapense & CBS 138008 & KJ869220 & - & KJ869163 & \\
\hline Neophaeosphaeria agaves & CBS 136429 & KF777227 & _ & KF777174 & \\
\hline Neophaeosphaeria filamentosa & CBS 102202 & GQ387577 & GQ387516 & JF740259 & \\
\hline Neophaeosphaeria filamentosa & CBS 102203 & JX681104 & - & - & \\
\hline Ophiosphaerella herpotricha & CBS 620.86 & DQ678062 & DQ678010 & KF498728 & \\
\hline Paraphoma radicina & CBS 111.79 & EU754191 & EU754092 & KF251172 & \\
\hline Phaeosphaeriaoryzae & CBS 110110 & KF251689 & KF251186 & GQ387530 & \\
\hline Pleospora herbarum & CBS 191.86 & GU238160 & GU238232 & NR_111243 & \\
\hline Pyrenochaeta cava & CBS 257.68 & EU754199 & EU754100 & JF740260 & \\
\hline Pyrenochaeta nobilis & CBS 407.76 & EU754206 & EU754107 & NR103598 & \\
\hline Pyrenochaetopsis leptospora & CBS 101635 & GQ387627 & GQ387566 & JF740262 & \\
\hline Pyrenochaetopsis pratorum & CBS 445.81 & GU238136 & GU238228 & JF740263 & \\
\hline Pyrenophor aphaeocomes & DAOM 222769 & JN940093 & JN940960 & JN943649 & \\
\hline
\end{tabular}




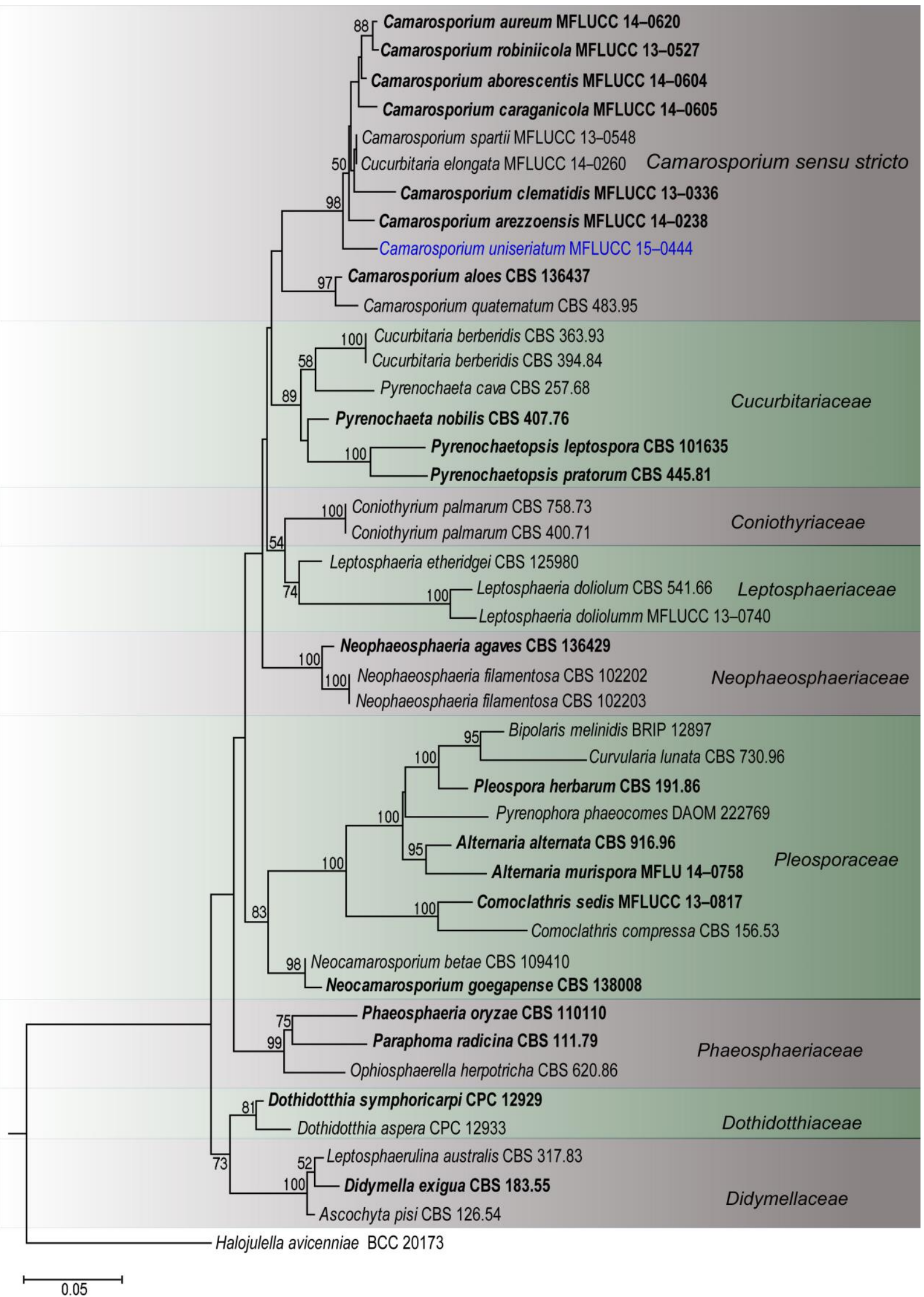

Fig. 1 - Maximum Likelihood (ML) tree from analysis of combined dataset of LSU, SSU and ITS sequence data of Pleosporinae, Pleosporales. Bootstrap support values equal or greater than 50\% are given above and below the nodes. The tree is rooted to Halojulella avicenniae(BCC 20173). Newly generated sequences are in blue. Type strains are in bold. 


\section{Results}

\section{Phylogenetic analysis}

The analysis was based on a combined LSU, SSU and ITS sequence data belonging to Pleosporinae, Pleosporales and the best scoring RAxML tree is shown in Fig. 1. The outgroup taxon, Halojulella avicenniae (BCC 20173) is clearly excluded from the other taxa. Species residing in Pleosporinae, Pleosporales were positioned on the tree, and represented Camarosporium sensu stricto and the families Coniothyriaceae, Cucurbitariaceae, Didymellaceae, Dothidotthiaceae, Leptosphaeriaceae, Neophaeosphaeriaceae, Phaeosphaeriaceae and Pleosporaceae. The new strain (MFLUCC 15-0444) clustered in a separate subclade in Camarosporium sensu stricto, with a strong bootstrap support $(98 \%)$.

\section{Taxonomy}

Camarosporium uniseriatum Thambugala, Bulgakov\& K.D. Hyde, nom. nov.

Fig. 2 Index Fungorum number: IF551991

Facesoffungi number: FoF 01964

Replaced synonym -Cucurbitaria celtidis Shear, Bull. Torrey bot. Club 29: 451 (1902); non

Camarosporium celtidis Ellis \& Everh., nec Gucevič

Etymology - In reference to single row arrangement of the ascospores in the asci

Necrotrophic or saprobic on twigs and thin branches of Celtis species. Sexual morph Ascomata 300-475 $\mu \mathrm{m}$ wide $\times 200-400 \mu \mathrm{m}$ high $(\bar{x}=390 \times 338 \mu \mathrm{m}, \mathrm{n}=10)$, black, semiimmersed, becoming erumpent, scattered, solitary to gregarious, globose to subglobose, coriaceous, rough or hairy, ostiolate. Ostiole central, short, ostiolar canal filled with hyaline to lightly pigmented pseudoparenchymatous cells. Peridium 30-60 $\mu \mathrm{m},(\bar{x}=45 \mu \mathrm{m}, \mathrm{n}=15)$ wide, comprising several layers, outer layers heavily pigmented, thick-walled, comprising blackish to dark brown cells of textura angularis, inner layers composed of hyaline, thin-walled cells of texturaangularis. Hamathecium comprising 1-3 $\mu \mathrm{m}$ wide, numerous, filamentous, septate, pseudoparaphyses. Asci $120-160 \times 12-15 \mu \mathrm{m}(\bar{x}=140 \times 13 \mu \mathrm{m}, \mathrm{n}=15), 8$-spored, bitunicate, fissitunicate, cylindrical, short-pedicellate, apex rounded, with an ocular chamber. Ascospores (13.5-) $15-26.5 \times 7-8.8(-10) \mu \mathrm{m}(\bar{x}=19.9 \times 7.9 \mu \mathrm{m}, \mathrm{n}=40)$, uniseriate, slightly overlapping, initially hyaline, becoming dark brown at maturity, ellipsoid, oblong to fusoid, straight, muriform, with 3-5 transverse septa, and 1-2(-3) longitudinal septa, deeply constricted at the central septum, with rounded or acute ends, smooth-walled, without a mucilaginous sheath. Asexual morph Undetermined.

Material examined - Russia, Rostov Region, Rostov-on-Don City, Botanical Garden of Southern Federal University, Higher Park, on twigs and branches of Celtis occidentalis L. (Cannabaceae) 5 March 2014, T. Bulgakov T 37 (MFLU 16-0469, reference specimen designated here), living culture MFLUCC 15-0444, ICMP.

Culture characteristics - Ascospores germinated on PDA at $25^{\circ} \mathrm{C}$ within $18 \mathrm{~h}$ and germ tubes produced from one or several septa. Colonies on PDA reaching $18 \mathrm{~mm}$ diam. after 4 days at $25{ }^{\circ} \mathrm{C}$, flat, circular, surface initially white, becoming olivaceous brown starting from the centre, after 2 weeks, reverse dark grey to black, surface smooth, with edge entire to slightly undulate.

Notes - Cucurbitaria celtidis was introduced by Shear (1902) from Celtis occidentalis in the USA. Our collection from Russia on the same host fits with the original description of $C$. celtidis (Shear 1902), but we observed 1-2(-3) longitudinal septa and a slightly size variation of ascospores, compared to those of in the original description (Table 2). We believe this is because the different geographical distributions as these two collections were made in different continents. Our collection of Cucurbitaria celtidis (MFLUCC 15-0444) forms a distant clade in Camarosporium sensu stricto, with a strong bootstrap support (98\%, Fig. 1). Therefore, we introduce Camarosporium uniseriatum to accommodate Cucurbitaria celtidis and we use the species epithet uniseriatum as this epithet celtidis and celtidicola are already used in the genus 


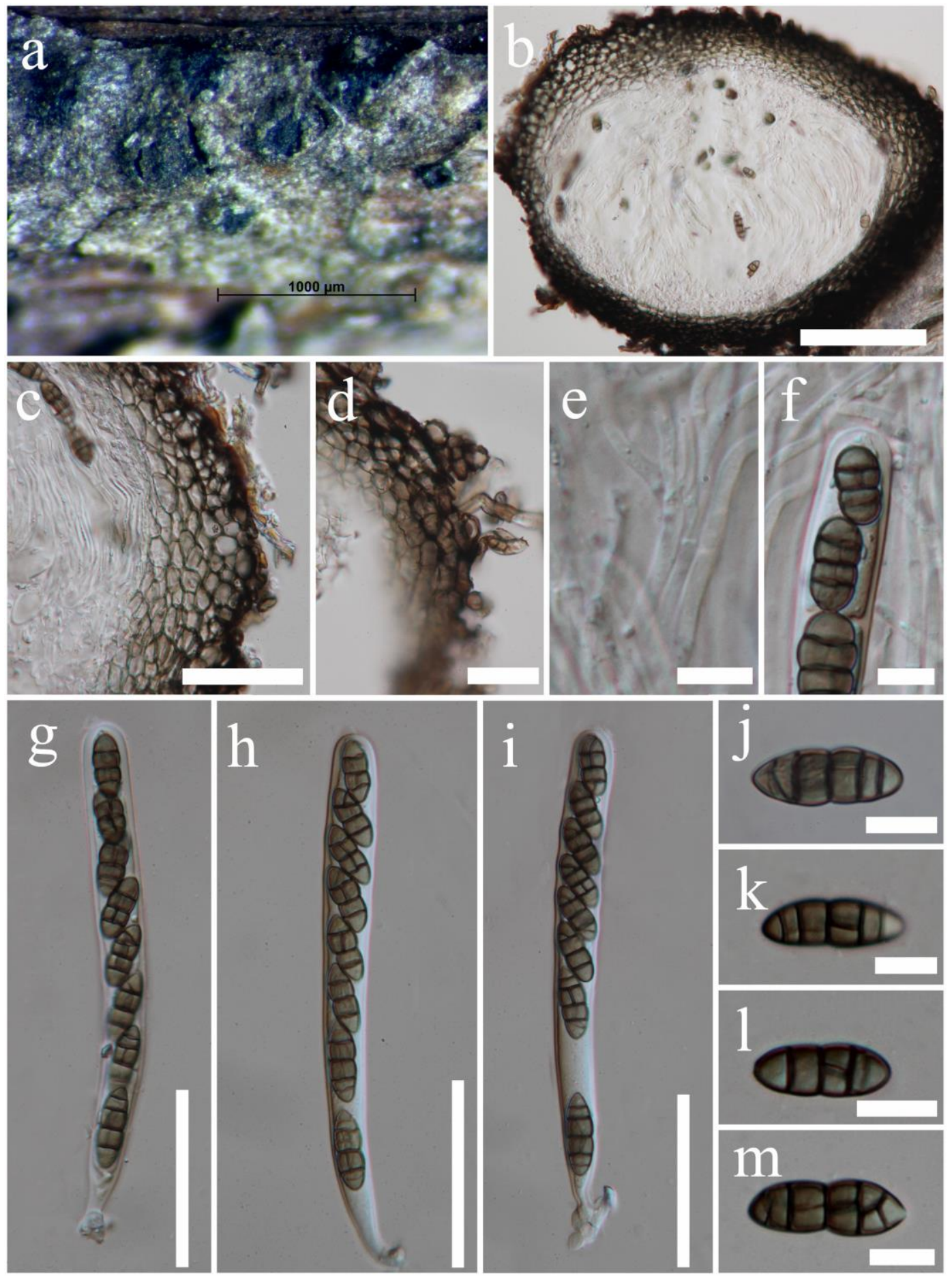

Fig. 2 - Camarosporium uniseriatum (MFLU 16-0469). a Appearance of ascomata on host surface. b Vertical section through ascoma. c, d Section through peridium (note light brown hyphae in d). E Pseudoparaphyses. f Apex of ascus g-i Asci. j-m Ascospores. Scale bars: $b=100 \mu \mathrm{m}, \mathrm{c}, \mathrm{g}-\mathrm{i}=50$ $\mu \mathrm{m}, \mathrm{d}=25 \mu \mathrm{m}$, e-f, $\mathrm{j}-\mathrm{m}=10 \mu \mathrm{m}$.

Camarosporium (C.celtidis Ellis \&Everh. and C. Celtidicola Gucevič). Camarosporium elongatum and $C$. arezzoensis, the sexual morphs reported for Camarosporium sensu stricto also differs from C. uniseriatum in having ellipsoidal ascospores with different number of longitudinal and transverse septa (Mirza 1968, Hyde et al. 2013, Tibpromma et al. 2016). Only an asexual morph was reported for Camarosporium celtidis and C. Celtidicola Gucevič (Ellis and Everhart 1894, Saccardo 1895, Gucevič 1959), therefore, these species need recollection and sequencing to confirm their placements. 
Table 2 Comparison of Camarosporium uniseriatum (Cucurbitaria celtidis) and collections in Shear (1902) from Celtis spp.

\begin{tabular}{|c|c|c|c|c|}
\hline & Ascomata $(\mu \mathrm{m})$ & $\operatorname{Asci}(\mu \mathrm{m})$ & Ascospores $(\mu \mathrm{m})$ & Host and locality \\
\hline This study & $\begin{array}{l}300-475 \text { wide } \times \\
200-400 \text { high }(\bar{x}= \\
390 \times 338)\end{array}$ & $\begin{array}{l}120-160 \times 12-15 \\
(\bar{x}=140 \times 13)\end{array}$ & $\begin{array}{l}(13.5-) 15-26.5 \times \\
7-8.8(-10) \quad(\bar{x}= \\
19.9 \times 7.9), 3-5 \\
\text { transverse septa } \\
\text { and } 1-2(-3) \\
\text { longitudinal septa }\end{array}$ & $\begin{array}{l}\text { C. occidentalis L., } \\
\text { Russia }\end{array}$ \\
\hline Shear (1902) & $350-500$ & $140-180 \times 16$ & $\begin{array}{l}24-27 \times 8 \mu \mathrm{m}, \\
3-5 \text { transverse } \\
\text { septa and } 1 \\
\text { longitudinal } \\
\text { septum }\end{array}$ & $\begin{array}{l}\text { C. occidentalis L., } \\
\text { USA }\end{array}$ \\
\hline
\end{tabular}

\section{Discussion}

The genus Camarosporium was treated in different families by various authors and has been reported as the asexual morph of Cucurbitariaceae, Phaeosphaeriaceae and Botryosphaeriales (Kirk et al. 2008, Wijayawardene et al. 2012, Zhang et al. 2012, Hyde et al. 2013). Wijayawardene et al. (2014b) established Camarosporium sensustricto in Pleosporinae, Pleosporales, based on phylogenetic analyses.In this paper, we showed Cucurbitaria celtidis to group in Camarosporium sensu stricto in Pleosporinae, Pleosporales and describe it as a novel sexual member in Camarosporium. However, the sequences of C. quaternatum in GenBank (CBS 483.95) are not from the type strain. There are no sequences data available for a number of Camarosporium and Cucurbitaria species. Therefore, re-collection, epitypification or reference specimens (Ariyawansa et al. 2014) with molecular data are essential to establish their placement according to the modern taxonomic concepts.

\section{Acknowledgments}

We would like to thank The Mushroom Research Foundation, Chiang Rai, Thailand and Guizhou Key Laboratory of Agricultural Biotechnology, Guizhou Academy of Agricultural Sciences, Guiyang, Guizhou Province, People's Republic of China for providing postgraduate scholarship support to Kasun M. Thambugala. Kevin D. Hyde thanks the Chinese Academy of Sciences, project number 2013T2S0030, for the award of Visiting Professorship for Senior International Scientists at Kunming Institute of Botany. Kasun M. Thambugala thanks Shaun Pennycook and Hiran A. Ariyawansa for helpful comments and advice on the manuscript.

\section{References}

Ariyawansa HA, Hawksworth DL, Hyde KD, Jones EBG, Maharachchikumbura SSN, Camporesi E, Manamgoda DS, Thambugala KM, Udayanga D, Daranagama A, Jayawardena R, Liu JK, McKenzie EHC, Phookamsak R, Senanayake IC, Shivas RG, Tian Q, Xu JC. 2014 Epitypification and neotypification: guidelines with appropriate and inappropriate examples. Fungal Diversity 69, 57-91. http://dx.doi.org/10.1007/s13225-014-0315-4

Ariyawansa HA, Thambugala KM, Manamgoda DS, Jayawardena R, Camporesi E, Boonmee S, Wanasinghe DN, Phookamsak R, Hongsanan S, Singtripop C, Chukeatirote E, Kang JC, Jones EBG, Hyde KD. 2015 - Towards a natural classification and backbone tree for Pleosporaceae. Fungal Diversity 71, 85-139. http://dx.doi.org/ 10.1007/s13225-015-0323-z

Carbone I, Kohn L. 1999 - A method for designing primer sets for speciation studies in filamentous ascomycetes. Mycologia 91, 553-556.

Chomnunti P, Hongsanan S, Aguirre-Hudson B, Tian Q, Peršoh D, Dhami MK, Alias AS, Xu JC, Liu XZ, Stadler M, Hyde KD. 2014 - The sooty moulds. Fungal Diversity 66, 1-36. http://dx.doi.org/10.1007/s13225-014-0278-5 
Doilom M, Liu JK, Jaklitsch WM, Ariyawansa H, Wijayawardene NN, Chukeatirote E, Zhang M, McKenzie E, Geml J, Voglmayr H, Hyde KD. 2013 - An outline of the family Cucurbitariaceae. Sydowia65(1), 167-192.

Ellis JB, Everhart BM. 1894 -New species of fungi from various localities. Proceedings of the Academy of Natural Sciences of Philadelphia. 46, 322-384.

Gucevič 1959 - Notulae Syst. Sect. Crypt. Inst. Bot. Acad. Sci. U.S.S.R. 12, 204.

Hall TA. 1999 - BioEdit: a user-friendly biological sequence alignment editor and analysis program for Windows 95/98/NT. In: Nucleic Acids Symposium Series, pp 95-98.

Hyde KD, Jones EBG, Liu JK, Ariyawansa H, Boehm E, Boonmee S, Braun U, Chomnunti P, Crous PW, Dai DQ, Diederich P, Dissanayake A, Doilom M, Doveri F, Hongsanan S, Jayawardena R, Lawrey JD, Li YM, Liu YX, Lücking R, Monka J, Muggia L, Nelsen MP, Pang KL, Phookamsak R, Senanayake IC, Shearer CA, Suetrong S, Tanaka K, Thambugala KM, Wijayawardene NN, Wikee S, Wu HX, Zhang Y, Begoña AH, Alias SA, Aptroot A, Bahkali AH, Bezerra JL, Bhat DJ, Camporesi E, Chukea E, Gueidan C, Hawksworth DL, Hirayama K, Hoog SD, Kang JK, Knudsen K, Li WJ, Li XH, Liu ZY, Mapook A, Mckenzie EHC, Miller AN, Mortimer PE, Phillips AJL, Raja HA, Scheuer C, Schumm F, Taylor JE, Tian Q, Tibpromma S, Wanasinghe DN, Wang Y, Xu JC, Yacharoen S, Yan JY, Zang M. 2013 - Families of Dothideomycetes. Fungal Diversity 63, 1-313. http://dx.doi.org/10.1007/s13225-013-0263-4

Index Fungorum 2016 - http://www.indexfungorum.org/names/names. asp, Accessed 25 January 2016.

Jayasiri SC, Hyde KD, Ariyawansa HA, Bhat J, Buyck B, Cai L, Dai YC, Abd-Elsalam KA, Ertz D, Hidayat I, Jeewon R, Jones EBG, Bahkali AH, Karunarathna SC, Liu JK, Luangsa-ard JJ, Lumbsch HT, Maharachchikumbura SSN, McKenzie EHC, Moncalvo, JM, Ghobad-Nejhad M, Nilsson H, Pang KA, Pereira OL, Phillips AJL, Raspé O, Rollins AW, Romero AI, Etayo J, Selçuk F, Stephenson SL, Suetrong S, Taylor JE, Tsui CKM, Vizzini A, AbdelWahab MA, Wen TC, Boonmee S, Dai DQ, Daranagama DA, Dissanayake AJ, Ekanayaka AH, Fryar SC, Hongsanan S, Jayawardena RS, Li WJ, Perera RH, Phookamsak R, de Silva NI, Thambugala KM, Tian Q, Wijayawardene NN, Zhao RL, Zhao Q, Kang JC, Promputtha I. 2015 - The Faces of Fungi database: fungal names linked with morphology, phylogeny and human impacts. Fungal Diversity 74(1), 3-18. http://dx.doi.org/10.1007/s13225-01503518-8

Kirk PM, Cannon PF, Minter DW, Staplers JA. 2008 - Dictionary of the Fungi 10th edn. CABI Bioscience, UK.

Liu JK, Hyde KD, Jones EBG, Ariyawansa HA, Bhat DJ, Boonmee S, Maharachchikumbura S, McKenzie EHC, Phookamsak R, Phukhamsakda C, Shenoy BD, Abdel-Wahab MA, Buyck B, Chen J, Chethana KWT, Singtripop C, Dai DQ, Dai YC, Daranagama DA, Dissanayake AJ, Doliom M, D'souza MJ, FanXL, Goonasekara ID, Hirayama K, Hongsanan S, Jayasiri SC, Jayawardena RS, Karunarathna SC, Li WJ, Mapook A, Norphanphoun C, Pang KL, Perera RH, Peršoh D, Pinruan U, Senanayake IC, Somrithipol S, Suetrong S, Tanaka K, Thambugala KM, Tian Q, Tibpromma S, Udayanga D, Wijayawardena NN, Wanasinghe D, Wisitrassameewong K, Abdel-Aziz FA, Adamčík S, Bahkali AH, Boonyuen N, Bulgakov T, Callac P, Chomnunti P, Greiner K, Hashimoto A, Hofstetter V, Kang JC, Lewis D, Li XH, Liu XX, Liu ZY, Matumura M, Mortimer PE, Rambold G, Randrianjohany E, Sato G, SriIndrasutdhi V, Tian CM, Verbeken A, von Brackel W, Wang Y, Wen TC, Xu JC, Yan JY, Zhao RL, Camporesi E. 2015 - Fungal diversity notes 1-110: taxonomic and phylogenetic contributions to fungal species. Fungal Diversity 72, 1-197. http://dx.doi.org/10.1007/s13225-015-0324-y

Miller MA, Pfeiffer W, Schwartz T. 2010 - "Creating the CIPRES Science Gateway for inference of large phylogenetic trees" in Proceedings of the Gateway Computing Environments Workshop (GCE), 14 Nov. 2010, New Orleans, LA pp 1-8. 
Mirza F. 1968 - Taxonomic investigations on the ascomycetous genus Cucurbitaria. Nova Hedwigia 16, 161-213.

Saccardo PA. 1895 - SyllogeFungorum XI: 537.

Schulzer S. 1870 - MykologischeBeiträge.Verhandlungen der Zoologisch-BotanischenGesellschaft Wien 20, 635-658.

Shear CL. 1902 - Mycological notes and new species. Bulletin of the Torrey Botanical Club 29, 449-457.

Stamatakis A, Hoover P, Rougemont J. 2008 - A rapid bootstrap algorithm for the RAxML web servers. Systematic biology 57, 758-771.

Tamura K, Peterson D, Peterson N, Stecher G, Nei M, Kumar S. 2011 - MEGA5: molecular evolutionary genetics analysis using maximum likelihood, evolutionary distance, and maximum parsimony methods. Molecular biology and evolution 28(10), 2731-2739.

Thambugala, KM, Hyde KD, Tanaka K, Tian Q, Wanasinghe DN, Ariyawansa H, Jayasiri SC, Boonmee S, Camporesi E, Hashimoto A, Hirayama K, Schumacher RK, Promputtha I, Liu ZY. 2015 - Towards a natural classification and backbone tree for Lophiostomataceae, Floricolaceae, and Amorosiaceaefam. nov. Fungal Diversity 74 (1):199-266. DOI 10.1007/s13225-015-0348-3

Tibpromma S, Wijayawardene NN, Manamgoda DS, Boonmee S, Wanasinghe DN, Camporesi E, Yang JB, Hyde KD. 2016 -Camarosporiumarezzoensis on Cytisus sp., an addition to sexual state of Camarosporiumsensustricto. Saudi Journal of Biological Sciences23(1), 18.http://dx.doi.org/10.1016/j.sjbs.2015.01.018

Vilgalys R, Hester M. 1990 - Rapid genetic identification and mapping of enzymatically amplified ribosomal DNA from several Cryptococcus species. Journal of bacteriology 172, 4238 4246.

White TJ, Bruns T, Lee S, Taylor JW. 1990 - Amplification and direct sequencing of fungal ribosomal RNA genes for phylogenetics. In: Innis MA, Gelfand DH, Sninsky JJ, White TJ. (eds) PCR protocols: a guide to methods and applications. New York Academic Press, pp 315-322

Wijayawardene DNN, McKenzie EHC, Hyde KD 2012- Towards incorporating anamorphic fungi in a natural classification-checklist and notes for 2011.Mycosphere 3(2), 157-228.

Wijayawardene NN, Hyde KD, Bhat DJ, Camporesi E, Schumacher RK, Chethana KWT, Wikee S, Bahkali AH, Wang Y. 2014a - Camarosporium-like species are polyphyletic in Pleosporales; introducing Paracamarosporium and Pseudocamarosporium gen. nov.in Montagnulaceae. $\quad$ Cryptogamiemycologie $\quad 35 \quad$ (2), 198.doi/10.7872/crym.v35.iss2.2014.177

Wijayawardene NN, Bhat DJ, Hyde KD, Camporesi E, Wikee S, Chethana KWT, Tangthirasunun N, Wang Y. 2014b - Camarosporium sensus tricto in Pleosporinae, Pleosporales with two new species. Phytotaxa 183 (1), 16-26. http://dx.doi.org/10.11646/phytotaxa.183.1.2

Wijayawardene NN, Hyde KD, Camporesi E, Bhat DJ, Goonasekara ID, Nadeeshan D, Camporesi E, Schumacher RK, Wang Y 2015-Additions to brown sporedcoelomycetous taxa in Massarinae, Pleosporales: introducing Phragmocamarosporium gen. nov. and Suttonomyces gen. nov. Cryptogam Mycol 36(2), 213-224.

Wijayawardene NN, Hyde KD, Wanasinghe DN, Papizadeh M, Goonasekara ID, Camporesi E, Bhat DJ, McKenzie EHC, Phillips AJL, Diederich P, Tanaka K, Li WJ, Tangthirasunun N, Phookamsak R, Dai DQ, Dissanayake AJ, Weerakoon G, Maharachchikumbura SSN, Hashimoto A, Matsumura M, Bahkali AH, Wang Y 2016-Taxonomy and phylogeny of dematiaceouscoelomycetes. Fungal Diversity (in press).

Zhang Y, Crous PW, Schoch CL, Hyde KD. 2012 - Pleosporales. Fungal Diversity 53, 1-221. DOI 10.1007/s13225-011-0117-x 